\title{
Elasticity of Natural Rubber Networks
}

\author{
P. H. Mott and C. M. Roland* \\ Chemistry Division, Code 6120, Naval Research Laboratory, Washington, D.C. 20375-5342 \\ Received February 6, 1996; Revised Manuscript Received J une 17, 1996 ${ }^{\circledR}$
}

\begin{abstract}
Stress, strain, and optical birefringence were measured for cross-linked natural rubber in compression and tension over a range of strains. The experimental data were compared to the constrainedjunction theory (Flory, P. J .; E rman, B. Macromolecules 1982, 15, 800) and to the constrained-chain theory (Kloczkowski, A.; Mark, J . E.; E rman, B. Macromolecules 1995, 28, 5089). These elasticity theories were found to fit both the stress and birefringence data quite well in tension. However, discrepancies arise when both compression and tension data are analyzed together. The differences between the models were minor, compared to their deviation from experiment.
\end{abstract}

\section{Introduction}

The study of cross-linked rubber offers unique insights into polymer physics because equilibrium data can be obtained. Much effort has been devoted to the development of theories able to predict the elastic behavior of networks. A proper assessment of elasticity theories requires measurements over a range of experimental variables, such as network structure, temperature, concentration, and deformation type, and ideally includes data beyond simply the strain dependence of the stress.

Particularly informative are stress measurements made in the transition region around $\lambda_{\|}=1$, where the stretch ratio, $\lambda_{\|}$, is the ratio of the deformed and undeformed sample lengths parallel to the applied force. Such experiments were carried out by Rivlin and Saunders ${ }^{1}$ on natural rubber networks; however, two different techniques were employed-inflation for $\lambda_{\|}<$ 1 and uniaxial extension for $\lambda_{\|}>1$-introducing artifacts into the data near $\lambda_{\| 1}=1$. Pak and Flory ${ }^{2}$ used a similar method for poly(dimethylsiloxane) (PDMS), obtaining data in both tension and compression, although the measurements exhibited substantial hysteresis, implying nonequilibrium. By using glued cylinders, Wolf ${ }^{3}$ obtained data on cross-linked natural rubber in both tension and compression; however, the latter were limited to $\lambda_{\|} \geq 0.88$. A problem therein was the inhomogeneous strains arising in uniaxially deformed cylinders with bonded ends. Erman and Flory ${ }^{4}$ also used a cylindrical geometry, obtaining data on PDMS for strains of about $10 \%$ in compression and tension. In both these studies, 3,4 the data were not corrected for the inhomogeneous strain.

While the strain dependence of the stress is the most common quantity used to assess elasticity theories, measurements of the optical birefringence are equally informative. The stress optical coefficient, $C$, defined as the ratio of the birefringence, $\Delta \mathrm{n}$, to the true stress, $\sigma$, is often taken to be a constant (Brewster's law). Actually, $C$ has a weak stress dependence and previous workers have used comparisons of calculated and experimentally determined values of the birefringence to assess elasticity theories. Experiments have been performed on natural rubber, ${ }^{5-7}$ PDMS, ${ }^{8,9}$ polybutadiene, ${ }^{10,11}$ and polyisoprene, ${ }^{11}$ but in all of these studies, the data were restricted to tension. To date, a study of birefringence of rubbers in compression has not been carried out. 1996.
In this paper, we describe measurements of both stress and birefringence on deproteinized natural rubber uniaxially deformed over the range $0.5<\lambda_{\|}<4$. Constitutive equations taken from the constraint theories of rubber elasticity were applied to the data.

\section{Background}

Early models of rubber elasticity describe two extremes in the relationship of stress to strain-a phantom network, comprised of volumeless chains able to pass through one another, ${ }^{12}$ and an affine network, whose chains are firmly embedded in a deformable continuum. ${ }^{13}$ For an incompressible rubber, the engineering stress for the phantom network is given by ${ }^{14,15}$

$$
\sigma_{\mathrm{ph}}=2\left(1-\frac{2}{\phi}\right) \mu \mathrm{RT}\left(\alpha-\alpha^{-2}\right)
$$

where $\phi$ is the cross-link functionality, $\mu$ the cross-link density, and RT has its usual significance. The elongation, $\alpha$, is related to the stretch ratio, $\lambda_{\|}$, by $\alpha=\lambda_{11}\left(\mathrm{~V}_{0} /\right.$ $\mathrm{V})^{1 / 3}$, where $\mathrm{V}_{0}$ and $\mathrm{V}$ are the initial and swollen volumes, respectively. All experiments herein were carried out on neat networks, whereby $\alpha=\lambda_{\|}$. Equation 1 assumes all chains are elastically active, which is strictly true only if the primary molecular weight is much larger than the molecular weight between crosslinks. ${ }^{16}$ The stress of an affine network, which does not depend on the cross-link functionality, is twice that given by eq 1 for $\phi=4$.

The most obvious failing of the classical theories is the deviation of experimental results from the predicted proportionality between engineering stress, $\sigma_{\text {eng, }}$ and the stretch function, $\left(\lambda_{\|}-\lambda_{\|}{ }^{-2}\right)$. This led to the development of phenomenol ogical al ternatives, the most widely used of which is the Mooney-Rivlin equation. ${ }^{14}$ The appeal of the Mooney-Rivlin approach is largely due to the ease with which the el astic constants can be determined by a linear regression of the reduced stress, $\sigma_{\text {eng }} /\left(\lambda_{\|}-\right.$ $\left.\lambda_{\|}{ }^{-2}\right)$ ), versus $1 / \lambda_{\|}$. However, such plots are often not linear, particularly for elastomers subjected to both tension and compression. 17,18

An important development in the theory of rubber elasticity was Flory's recognition of the role of intermolecular interactions in modifying the stress in a straindependent manner. This idea was incorporated into a constrained-junction (CJ ) model, 19 in which the engineering stress is given by

$$
\sigma_{\mathrm{CJ}}=\sigma_{\mathrm{ph}}\left[1+\frac{2}{\phi-2} \frac{\alpha \mathrm{K}\left(\lambda_{\|}{ }^{2}\right)-\alpha^{-2} \mathrm{~K}\left(\lambda_{\perp}{ }^{2}\right)}{\alpha-\alpha^{-2}}\right]
$$


where $\lambda_{\perp}$ is the stretch ratio perpendicular to the applied load $\left(=\lambda_{\|}-1 / 2\right.$ for uniaxial deformation assuming incompressibility). The function $\mathrm{K}\left(\lambda^{2}\right)$ is defined as

$$
K\left(\lambda^{2}\right)=\frac{B \dot{B}}{B+1}+\frac{D \dot{D}}{D+1}
$$

where

$$
\begin{gathered}
\mathrm{B}\left(\lambda^{2}\right)=\frac{\kappa^{2}\left(\lambda^{2}-1\right)}{\left(\lambda^{2}+\kappa\right)^{2}} \\
\dot{\mathrm{B}}\left(\lambda^{2}\right) \equiv \frac{\partial \mathrm{B}\left(\lambda^{2}\right)}{\partial\left(\lambda^{2}\right)}=\mathrm{B}\left(\lambda^{2}\right)\left[\frac{1}{\lambda^{2}-1}-\frac{2}{\lambda^{2}+\kappa}\right] \\
\mathrm{D}\left(\lambda^{2}\right)=\frac{\lambda^{2}}{\kappa} \mathrm{B}\left(\lambda^{2}\right)
\end{gathered}
$$

and

$$
\dot{\mathrm{D}}\left(\lambda^{2}\right) \equiv \frac{\partial \mathrm{D}\left(\lambda^{2}\right)}{\partial\left(\lambda^{2}\right)}=\frac{1}{\kappa}\left[\lambda^{2} \dot{\mathrm{B}}\left(\lambda^{2}\right)+\mathrm{B}\left(\lambda^{2}\right)\right]
$$

The parameter $\kappa$ in eqs $4-7$ is a measure of the severity of the topological constraints. Equation 3 reduces to the phantom and affine results for $\kappa=0$ and $\kappa=\infty$, respectively.

Subsequently, Flory and Erman ${ }^{20}$ extended the CJ model, introducing an additional parameter, $\zeta(\geq 0)$, to allow for departures from affine distortion, as occasioned, for example, by inhomogeneities in the network structure. A large value of $\zeta$ implies that the constraints are alleviated by extension of the network more rapidly than if the domains deformed affinely. In this extended constrained junction (ECJ ) model, $\mathrm{K}\left(\lambda^{2}\right)$ is still given by eq 3 , but the functions $B\left(\lambda^{2}\right)$ and $D\left(\lambda^{2}\right)$ become

$$
\mathrm{B}\left(\lambda^{2}\right)=\frac{\kappa^{2}\left[\lambda^{2}-1-\lambda^{2} \zeta(\lambda-1)\right]}{\left[\lambda^{2}+\kappa+\kappa \lambda^{2}(\lambda-1)\right]^{2}}
$$

and

$$
\mathrm{D}\left(\lambda^{2}\right)=\lambda^{2}\left[\frac{1}{\kappa}+\zeta(\lambda-1)\right] \mathrm{B}\left(\lambda^{2}\right)
$$

The derivatives $\dot{\mathbf{B}} \equiv \partial \mathbf{B} / \partial \lambda^{2}$ and $\dot{\mathbf{D}} \equiv \partial \mathrm{D} / \partial \lambda^{2}$ are not shown. Substitution of these quantities into eq 2 yields the engineering stress. The original CJ model is recovered when $\xi=0$.

In both the CJ and ECJ models, the effect of the constraints is assumed to be exerted directly on the network junctions. An alternative is to locate their effect at the chain's center of gravity. ${ }^{21,22}$ This idea has been generalized to al low the constraints to act all along the chain, the so-called continuously constrained chain (CC) model. ${ }^{23}$ The expression for the engineering stress in the CC model is

$$
\begin{aligned}
& \sigma_{\mathrm{CC}}= \\
& \sigma_{\mathrm{ph}}\left[1+\frac{\phi}{\phi-2} \int_{0}^{1} \mathrm{~W}(\Theta) \frac{\alpha \mathrm{K}\left(\lambda_{\|}{ }^{2}\right)-\alpha^{-2} \mathrm{~K}\left(\lambda_{\perp}{ }^{2}\right)}{\alpha-\alpha^{-2}} \mathrm{~d} \Theta\right]
\end{aligned}
$$

\begin{tabular}{|c|c|c|c|c|c|c|c|}
\hline \multirow[b]{2}{*}{ network } & \multirow{2}{*}{$\begin{array}{c}\text { dicumyl } \\
\text { peroxide (phr) }\end{array}$} & \multirow[b]{2}{*}{$\mu^{\mathrm{a}}$} & \multicolumn{2}{|c|}{ CC Model } & \multicolumn{3}{|c|}{ ECJ Model } \\
\hline & & & $\mu$ & $\kappa$ & $\mu$ & $\kappa$ & $\xi$ \\
\hline $\begin{array}{l}\text { NR-1 } \\
\text { NR-2 }\end{array}$ & $\begin{array}{l}1.0 \\
2.0\end{array}$ & $\begin{array}{l}33 \\
66\end{array}$ & $\begin{array}{r}65 \\
103\end{array}$ & $\begin{array}{l}3 \\
3\end{array}$ & $\begin{array}{r}81 \\
132\end{array}$ & $\begin{array}{l}6 \\
6\end{array}$ & $\begin{array}{l}0.2 \\
0.2\end{array}$ \\
\hline
\end{tabular}

where $K\left(\lambda^{2}\right)$ is again given by eq 3. $W(\Theta)$ is a distribution function describing the effectiveness of the constraints at each point along the chain, and $\Theta$, which varies from 0 to 1 , is the relative distance of a point on the chain from the junction site. If the strength of the
Table 1. Rubber Networks and Fitting Parameters

a Cross-link density $\left(\mathrm{mol} / \mathrm{m}^{3}\right)$ calculated assuming depletion of the peroxide and tetrafunctional junctions.

constraints is taken to be constant along the chain, $\mathrm{W}(\Theta)$ equals unity. ${ }^{23}$ Contrary to the earlier models, in which $\kappa$ is constant, for the CC model the constraint parameter $\kappa$ embedded in eq 10 is a function of position along the chain,

$$
\kappa(\Theta)=\kappa\left[1+\frac{(\Theta-2)^{2} \Theta(1-\Theta)}{\phi-1}\right]
$$

The constraint parameter $\kappa(\Theta)$ replaces the $\kappa$ in eqs $4-7$.

While the stress-optical coefficient remains constant through moderate strains, deviations from Brewster's law occur for larger deformations. Thus, the strain dependence of the birefringence $\Delta \mathrm{n}$ provides a useful quantity to independently assess the validity of a constitutive equation. According to the ECJ model, 8

$$
\Delta \mathrm{n}=\sigma \mathrm{C} \frac{1+\frac{\mathrm{B}\left(\lambda_{\|}{ }^{2}\right)-\mathrm{B}\left(\lambda_{\perp}{ }^{2}\right)+\mathrm{bD}\left(\lambda_{\|}{ }^{2}\right)-\mathrm{bD}\left(\lambda_{\perp}{ }^{2}\right)}{(\phi / 2-1)\left(\mathrm{V} N_{0}\right)^{2 / 3}\left(\alpha^{2}-\alpha^{-1}\right)}}{1+\frac{\lambda_{\|}{ }^{2} \mathrm{~K}\left(\lambda_{\|}{ }^{2}\right)-\lambda_{\perp}{ }^{2} \mathrm{~K}\left(\lambda_{\perp}{ }^{2}\right)}{(\phi / 2-1)\left(\mathrm{V} N_{0}\right)^{2 / 3}\left(\alpha^{2}-\alpha^{-1}\right)}}
$$

where $C$ is now the stress-optical coefficient measured at infinitesimal strain, and $b$ is an adjustable parameter $(0 \leq b \leq 1)$ reflecting the effect of orientation of the domains of constraint. The functions $K\left(\lambda^{2}\right), B\left(\lambda^{2}\right)$, and $D\left(\lambda^{2}\right)$ are given by eqs 3,8 , and 9 , respectively. The CC model makes analogous predictions for $\Delta n^{9,24,25}$

\section{Experimental Section}

The polymer was deproteinized natural rubber (SMR-S from H. A. Astlett Co.), which is cis-1,4-polyisoprene. Removal of the proteins significantly enhances transparency in comparison to conventional Hevea brasiliensis. The cross-linking agent, dicumyl peroxide, was mixed into the rubber using a two-roll mill (Table 1). Both films $(65 \times 13 \times 1.6 \mathrm{~mm})$ and cylinders (12.2 mm diameter $\times 17.8 \mathrm{~mm}$ high) were prepared by compression molding for $30 \mathrm{~min}$ at $160^{\circ} \mathrm{C}$. The cross-link density calculated for the two networks, assuming depletion of the peroxide, is listed in Table 1.

The apparatus used to obtain compression data on the rubber cylinders is depicted in Figure 1. The cylinder ends were bonded to aluminum plates with cyanoacrylate adhesive. Calibrated weights were applied, and the change in sample length measured using a linear voltage differential transducer. Although bonding of the cylinder ends to the confining plates allows both tension and compression data to be obtained, it also gives rise to additional stress, due to shear forces acting on the cylinder ends. ${ }^{26}$ Correcting for the resulting barreling (or hourglass shape if $\alpha>1$ ) gives ${ }^{27}$

$$
\sigma_{\text {eng }}=\frac{\sigma_{\text {app }}}{1+\frac{\mathrm{R}_{0}^{2}}{2 \mathrm{H}_{0}^{2}}}
$$

where $\mathrm{R}_{0}$ and $\mathrm{H}_{0}$ are the initial sample radius and height, respectively, and $\sigma_{\mathrm{app}}$, the apparent stress, is the ratio of the applied force to the initial cross-sectional area of the cylinder. The corrected engineering stress $\sigma_{\text {eng }}$ given by eq 13 corresponds to the engineering stress that would be observed in a homogeneously-strained cylinder, deformed uniaxially. A 


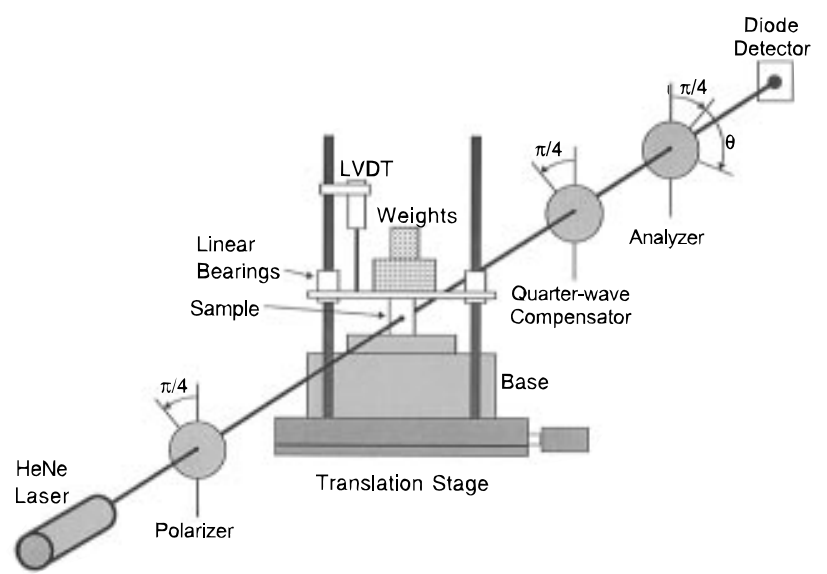

Figure 1. Schematic of the apparatus used for simultaneous measurement of the force and birefringence of rubber cylinders in uniaxial deformation.

comprehensive experimental assessment of eq 13 was recently carried out, 28 involving a comparison of stress, strain, and birefringence data obtained from bonded cylinders of varying aspect ratio as well as from nonbonded samples. This investigation demonstrated that eq 13 corrects the experimental data to an accuracy of better than $5 \%$ over substantial ranges of strain. ${ }^{28}$ Thus, any residual error due to bonding of the cylinder ends is less than the cumulative error intrinsic to mechanical experiments (e.g., reproducing the cross-link density, attaining mechanical equilibrium, etc.). This cumulative error we judge to be no more than $10 \%$.

Extension measurements were carried out by two methods. To ensure an absence of systematic error between the compression and tension experiments, the cylindrical samples were extended while in the compression geometry by means of a screw pulling on the loading stage; this yielded tension data through $\lambda=1.8$. Additional experiments to much higher extension were performed using films. Weights were attached to the samples, and the resulting strain determined from displacement of fiducial marks, measured using a cathetometer.

The birefringence was determined from the rotation, $\theta$, of an analyzer polarizing filter, as shown in Figure 1 . The birefringence is given by 29

$$
\Delta \mathrm{n}=\frac{\theta}{\pi} \frac{\Lambda}{\mathrm{l}}
$$

where $\Lambda=632.8 \mathrm{~nm}$ for $\mathrm{He}-\mathrm{Ne}$ laser radiation. The rotation of the analyzer reached values as high as $40 \pi$ for the highest compressive strains. The path length of the beam through the sample, I, was deduced for the extended strips by assuming incompressibility. For the cylinders, I corresponds to the maximum (compression) or minimum (extension) diameter of the deformed cylinder. This was measured by translating the deformed cylinder horizontally and identifying the outer surfaces with the laser beam. Parenthetically, we note that the usual assumption that compressed cylinders exhibit a parabolic profile is incorrect. ${ }^{28}$

All experimental results herein correspond to room temperature and mechanical equilibrium. A minimum of $6 \mathrm{~h}$ el apsed between applying the load and measuring the strain and the birefringence; thus, no more than two measurements could be obtained per day. Furthermore, to verify the absence of hysteresis, alternately increasing and decreasing changes in the load were made.

\section{Results and Discussion}

In Figure 2 the engineering stress versus stretch ratio for the natural rubber networks is displayed, after correction for the effect of inhomogeneous strain in the bonded cylinders (eq 13). Concerning the shape of the stress-strain curves in Figure 2, three observations can be made, all having been previously established for rubbery networks. First, the slopes of the curves

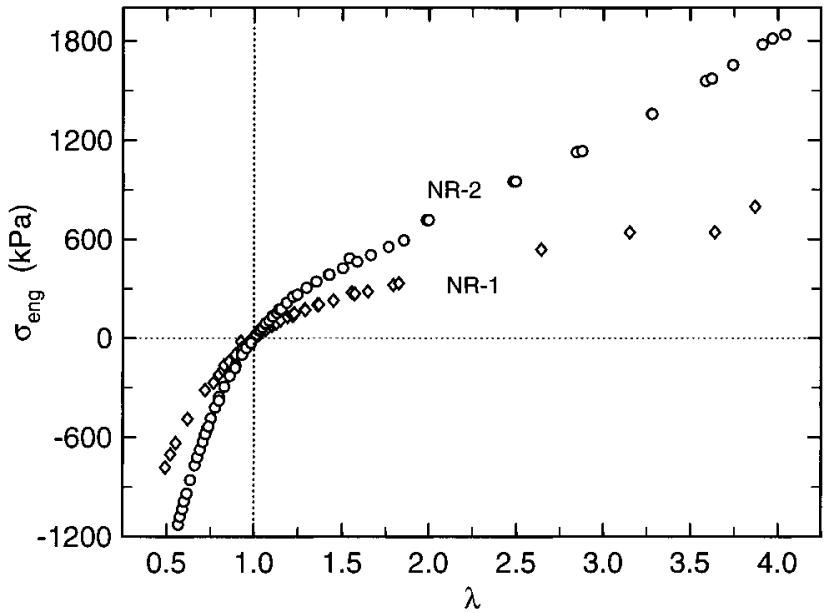

Figure 2. Engineering stress versus stretch ratio for the peroxide-cross-linked, deproteinized natural rubbers.

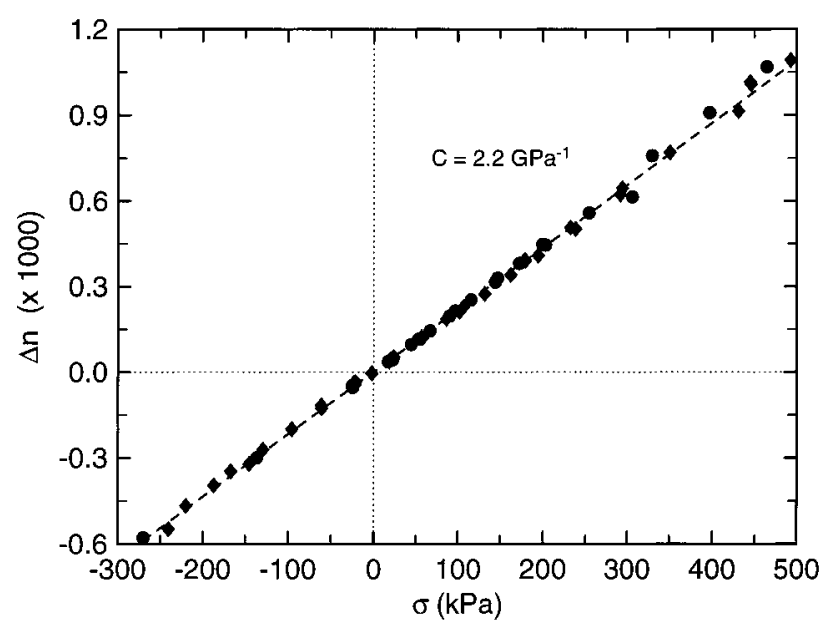

Figure 3. Optical birefringence as a function of true stress for NR-1 $(\diamond)$ and NR-2 $(\bullet)$ over the range $0.8 \leq \lambda_{\|} \leq 1.6$. Linear regression yields the indicated value for the stress optical coefficient C.

decrease as the material is taken from compression into tension. This lower modulus, as well as the reduced strain dependency of the modulus in compression, represents a classic failing of the most popular equation of state for elastomers, the Mooney-Rivlin expression. ${ }^{17,18}$ Second, the data in Figure 2 are continuous through $\lambda=1$. Although unsurprising, experimental data demonstrating this fact are relatively scarce. ${ }^{2,4,30,31}$ Third, at the highest tensile strains, the stress for the more cross-linked NR-2 shows an upturn, reflecting strain-induced crystallization. ${ }^{32-35} \mathrm{Natural}$ rubber has an unusual capacity for orientational crystallization, which underlies much of its commercial utility. ${ }^{36}$ Of course, once crystallization commences, rubber elasticity theories are no longer applicable.

In Figure 3 we plot the bi refringence measured at the center of the cylinders versus the true stress for $0.8 \leq$ $\lambda_{\|} \leq 1.6$. Over this limited range of strains, the stressoptical coefficient is constant to within the experimental error. Neglecting any weak dependence of C on crosslink density, ${ }^{14,28}$ we combine the data for NR-1 and NR-2 to obtain $\mathrm{C}=2.2 \mathrm{GPa}^{-1}$. This result is about $5 \%$ higher than the value reported for a natural rubber network of comparable cross-link density, but from which the proteins had not been removed. ${ }^{6}$

To compare the experimental stress-strain data to the constitutive models described above, the reduced stress, $\sigma_{\text {eng }} /\left(\lambda_{\|}-\lambda_{\|}{ }^{-2}\right)$, is plotted against $1 / \lambda_{\|}$. The data 


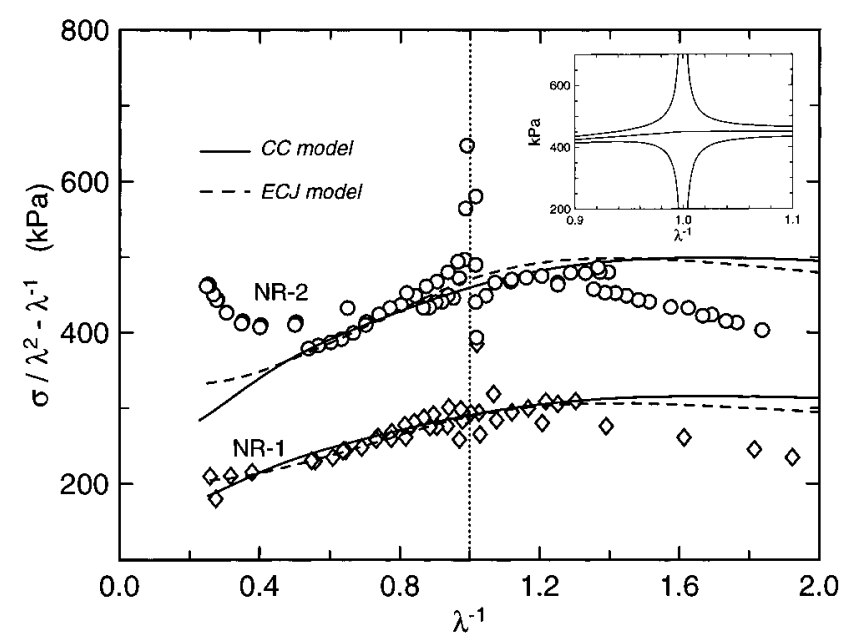

Figure 4. Reduced force versus reciprocal of the stretch ratio for the two natural rubber networks, al ong with the best fits to the ECJ theory (eqs 2, 8, and 9) and to the CC theory (eqs 10 and 11). The inset shows the experimental uncertainty in the vicinity of $\lambda_{\|}{ }^{-1}=1$.

from Figure 2 are displayed in this "Mooney" form in Figure 4. F or values near $\lambda_{\|}{ }^{-1}=1$, the reduced stress is extremely sensitive to errors in length. An error analysis ${ }^{37}$ carried out to determine this uncertainty is shown as an inset to Figure 4. Thus, extreme scatter of the data in the range $0.987 \leq \lambda_{\|}^{-1} \leq 1.02$ is expected. Interestingly, these deviations are positive for tension and negative for compression. This can perhaps be ascribed to systematic error (e.g., friction from the bearings in the support stage in Figure 1). However, more accurate, small-strain experiments carried out on natural rubber in both tension and compression re vealed very similar deviations in the reduced stress, which the authors did not attribute to experimental error ${ }^{30}$ Such behavior is not anticipated by any rubber elasticity theory, and perhaps deserves more careful study.

At moderate strains, the data in Figure 4 exhibit a maximum, roughly in the vicinity of $\lambda_{\|}^{-1}=1.2$, in accord with previous results on PDMS.2,4 At higher strains, particularly for the more cross-linked NR-2, straininduced crystallization ${ }^{35}$ causes a marked curvature in the experimental points. The curvature is the result of two competing effects: at the onset of crystallization, some reduction in the reduced stress occurs, since the formation of crystals reduces the number of elastically active chains. Eventually at higher strains, however, crystallinity raises the stress, because the crystallites serve to increase the effective cross-link density of the network. 33

We can compare the present results to the network theories described above. For the continuously constrained-chain theory (eqs 10 and 11), the distribution of constraints along the chain is taken to be uniform (i.e., $W(\Theta)=1$ ), with the cross-links assumed to be tetrafunctional $(\phi=4)$, as reputed for peroxide-crosslinked natural rubber. ${ }^{38}$ Thus, the only adjustable parameters are the cross-link density $\mu$ and the constraint parameter $\kappa$. In fitting the CC model to the NR-2 data, we ignored the highest strains, since these points were influenced by crystallization. The solid lines in Figure 4 represent fitting to the $C C$ model, with the values for the parameters $\mu$ and $\kappa$ listed in Table 1 . The figure shows that in extension $\left(\lambda_{\|}{ }^{-1}<1\right)$, the model describes the experimental data for both networks quite well; the difference between the calculated and measured results is less than the experimental error.

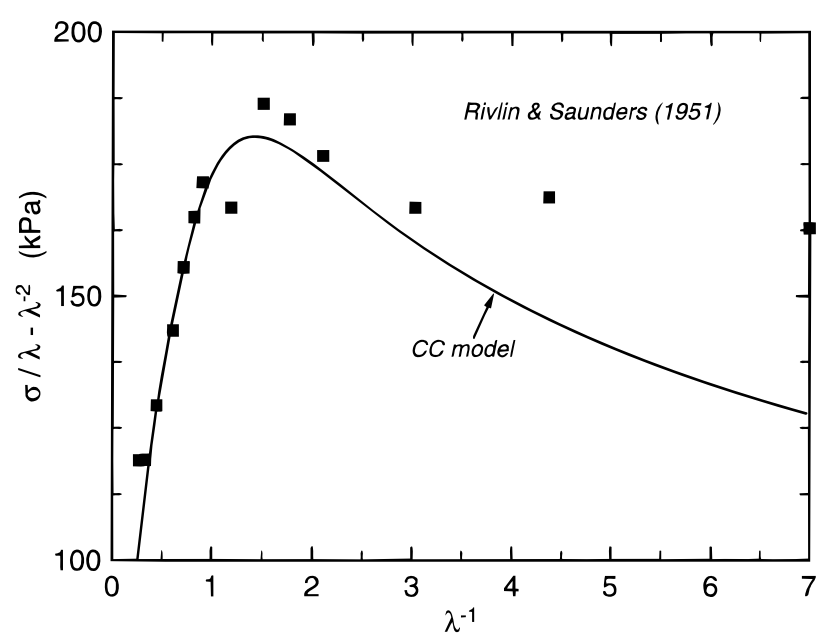

Figure 5. Reduced force versus inverse stretch ratio for sulfur-cross-linked natural rubber. The solid line was calculated from the CC theory (eqs 10 and 11 ).

H owever, the CC theory does not give good agreement with the data for either network in compression $\left(\lambda_{\|}{ }^{-1}>\right.$ 1 ); the reduced stress (modulus) decreases more strongly with compressive strain than predicted. Any modification of $\kappa$ to improve the agreement in compression causes the fitting for the tension data to deteriorate. This lack of a good fit over the entire range of deformation introduces some uncertainty in the parameters $\mu$ and $\kappa$ (Table 1 ), and their interpretation in terms of the details of the network structure is problematic.

An alternative to the CC model is the extended constrained-junction model (eqs 2, 8, and 9), in which the constraints on the chain fluctuations act only on the cross-links. Our interest in applying the ECJ model is whether the extra parameter, $\zeta$, provides for improved agreement with experiment. The dashed lines in Figure 4 show the results of fitting the ECJ model, with the values for the fit variables, $\mu, \kappa$, and $\xi$, listed in Table 1. Again, the imperfect quality of the fit confers some uncertainty to the value of the fitting parameters. N ote that differences between the CC and ECJ models are minor in comparison to their deviation from the experimental compression data.

The limited success of the models in describing both tension and compression data can be compared to the results of fitting previously published measurements on sulfur-vulcanized natural rubber. ${ }^{1}$ Unfortunately, there are some problems with this data. Unless the crosslinks are monosulfidic, sulfur vulcanization produces networks which are mechanically labile. In fact, a hint of such is evident in the authors' comment ${ }^{1}$ that consistent results (without hysteresis) required that only load-increasing experiments be conducted. This study also relied on two different experimental techniques-extension of strips for $\alpha>1$ and inflation of sheets for $\alpha<1$-which can be a source of error, particularly near $\alpha=1$.

Shown in Figure 5 are the results of fitting the data for the sulfur-cured networks ${ }^{1}$ to the CC model (eqs 10 and 11). Agreement between experiment and theory is again found only for extension. We also note that, consistent with our findings, this data was previously shown to conform to the original constrained junction model (i.e., $\zeta=0$ ) only for tension. ${ }^{2}$

While in Figure 3 the stress-optical coefficient $C$ was determined from the proportionality between the birefringence $\Delta \mathrm{n}$ and the true stress $\sigma$, deviations from linearity were notable at higher strains. In Figure 6, the birefringence $\Delta \mathrm{n}$ from the two networks is plotted 


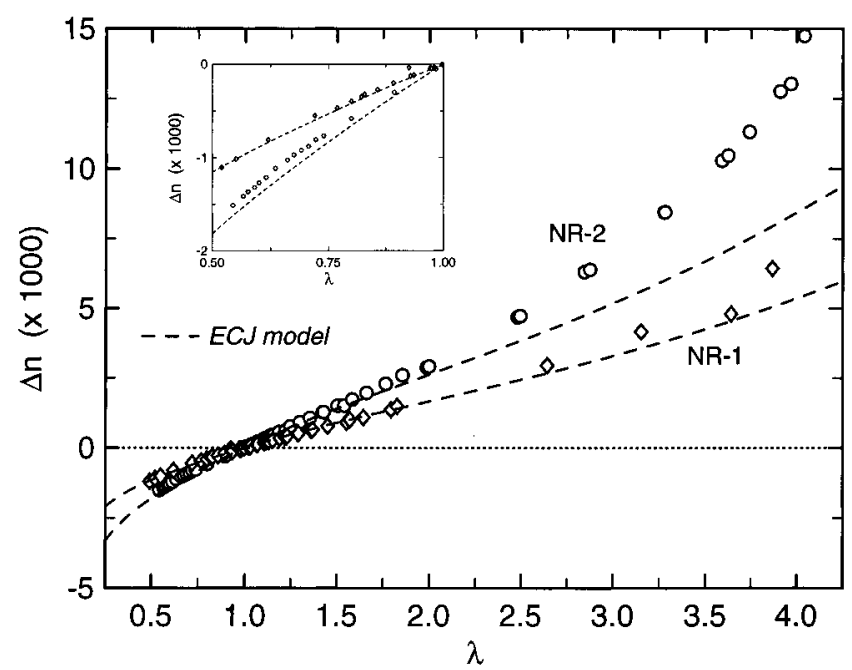

Figure 6. Optical birefringence as a function of strain for the two natural rubber networks, along with the curve calculated using eq 12. The deviations at high extension are due, at least in part, to orientational crystallization.

against $\lambda_{\|}$, al ong with the best fit of the data to the ECJ model (eq 12), with $b=0$. Given that the stress is only roughly described by the elasticity models for compression $\left(\lambda_{\|}<1\right)$, we cannot expect better success with regard to the birefringence. There is an additional complication from crystallization, apparent in Figure 4, but more evident in the birefringence data. Contrary to its effect on stress, crystallinity al ways augments the birefringence, ${ }^{31}$ and thus it is a sensitive means to detect its onset. This effect is seen in Figure 6 in the underestimation of $\Delta \mathrm{n}$ by the fitted ECJ model, beginning at moderate extensions $\left(\lambda_{\|}>2\right)$. At the highest extensions $\left(\lambda_{\|}>3.5\right)$, Figure 6 shows the birefringence increasing dramatically, corresponding to an order of magnitude increase in the stress-optical coefficient C.

\section{Summary}

The elasticity models of Flory and their extensions by various workers were assessed over a range of strains, including both compression and tension. We find that the constraint models describe stress, strain, and birefringence data on natural rubber networks quite well in tension; however, the agreement is unsatisfactory when both compression and tension data are considered together. The mediocre fit of theory to experiment was most evident when the reduced stress was analyzed. If consideration is restricted to the more conventional engineering stress-strain, the constraint models would suffice for many practical applications.

There are, of course, other rubber elasticity models, which have not been examined herein. Some ${ }^{39-42}$ are in many respects similar to the constraint models. Others have a more phenomenological basis ${ }^{1,31,43-46}$ and thus lack the intuitive clarity of the constraint theories. Our focus on the constraint theories reflects their widespread usage. Be that as it may, it is evident that further developments are required for a quantitative characterization of the behavior of rubbery networks. It is likely that contributions in this regard can be realized from investigations of network dynamics. 41,42,47-54

Acknowledgment. This work was supported by the Office of Naval Research. P.H.M. expresses his gratitude for a National Research Council/Naval Research Laboratory postdoctoral fellowship.

\section{References and Notes}

(1) Rivlin, R. S.; Saunders, D. W. Philos. Trans. R. Soc. London, Ser. A 1951, 243, 251.
(2) Pak, H.; Flory, P. J. J . Polym. Sci., Polym. Phys. Ed. 1979, $17,1845$.

(3) Wolf, F. P. Polymer 1972, 13, 347.

(4) Erman, B.; Flory, P. J . J . Polym. Sci., Polym. Phys. Ed. 1978, 16, 1115.

(5) Treloar, L. R. G. Trans. Faraday Soc. 1947, 43, 284.

(6) Saunders, D. W. Trans. Faraday Soc. 1956, 52, 1414.

(7) Smith, K. J .; Puett, D. J . Appl. Phys. 1966, 37, 346.

(8) Erman, B.; Flory, P. J . Macromolecules 1983, 16, 1601, 1607.

(9) Neaffer, R. O.; Galiatsatos, V. Macromolecules 1993, 26, 4013.

(10) Fukuda, M.; Wilkes, G. L.; Stein, R. S. J . Polym. Sci., Part A-2 1971, 9, 1417.

(11) Ishikawa, T.; Nagai, K.J . Polym. Sci., Part A-2 1969, 7, 1123; Polym. J. 1970, 1, 116.

(12) J ames, H. M.; Guth, E. J . J . Chem. Phys. 1947, 15, 669; 1953, 21, 1039.

(13) Kuhn, W. J . Polym. Sci. 1946, 1, 380.

(14) Treloar, L. R. G. ThePhysics of Rubber Elasticity; Clarendon Press: Oxford, U.K., 1975.

(15) Flory, P. J . Proc. R. Soc. London, Ser. A 1976, 351, 351.

(16) Flory, P. J . Macromolecules 1982, 15, 99.

(17) Rivlin, R. S. Rubber Chem.Technol. 1992, 65, G51.

(18) Flory, P. J . Polymer 1979, 20, 1317.

(19) Flory, P. J . J . Chem. Phys. 1977, 66, 5720.

(20) Flory, P. J .; Erman, B. Macromol ecules 1982, 15, 800.

(21) Erman, B.; Monnerie, L. Macromolecules 1989, 22, 3342.

(22) Fontaine, F.; Morland, C.; Loel, C.; Monnerie, L.; Erman, B. Macromol ecules 1989, 22, 3348.

(23) Kloczkowski, A.; Mark, J. E.; Erman, B. Macromolecules 1995, 28, 5089.

(24) Galiatsatos, V. Macromolecules 1990, 23, 3817.

(25) Kloczkowski, A.; Mark, J . E.; Erman, B. Comput. Polym. Sci. 1995, 5, 37.

(26) Gent, A. N.; Henry, R. L.; Roxbury, M. L. J . Appl. Mech. 1974, $41,855$.

(27) Gent, A. N. Science and Technology of Rubber; Mark, J . E., Erman, B., Eirich, F. R., Eds.; Academic Press: San Diego, 1994; Chapter 1.

(28) Mott, P. H.; Roland, C. M. Rubber Chem. Technol. 1995, 68, 739.

(29) Riande, E.; Saiz, E. Dipole Moments and Birefringence of Polymers; Prentice-Hall: Englewood Cliffs, NJ , 1992.

(30) McKenna, G. B.; Zappas, L. J . Polymer 1983, 24, 1502.

(31) Treloar, L. R. G. Rep. Prog. Phys. 1973, 36, 755.

(32) Gehman, S. D.; Field, J . E. Rubber Chem. Technol. 1939, 12 706; 1944, 17, 640

(33) Mark, J. E. Polym. Eng. Sci. 1979, 19, 254.

(34) Amram, B.; Bokobza, L.; Queslel, J . P.; Monnerie, L. Polymer 1986, 27, 877.

(35) Roland, C. M.; Warzel, M. L. Rubber Chem. Technol. 1990, 63, 285.

(36) Allegra, G.; Bruzzone, M. Macromolecules 1983, 16, 1167.

(37) Holman, J. P.; Gajda, W. J . Experimental Methods for Engineers, 3rd ed.; McGraw-Hill: New York, 1978; Chapter 3.

(38) Coran, A. Y. Science and Technol ogy of Rubber; Mark, J . E., Erman, B., Eirich, F. R., Eds.; Academic Press: San Diego, 1994; Chapter 7.

(39) Ronca, G.; Allegra, G. J . Chem. Phys. 1975, 63, 4990.

(40) Vilgis, T. A.; Erman, B. Macromolecules 1993, 26, 6657.

(41) Edwards, S. F.; Vilgis, T. Rep. Prog. Phys. 1988, 51, 243.

(42) Edwards, S.; Vilgis, T. Polymer 1986, 27, 483.

(43) Arruda, E. M.; Przybylo, P. A. Polym. Eng. Sci. 1995, 35, 395.

(44) Martin, G. M.; Roth, F. L.; Stiehler, R. D. Trans. Inst. Rubber Ind. 1956, 32, 189.

(45) Blatz, P. J .; Sharda, S. C.; Tschoegl, N. W. Trans. Soc. Rheol. 1974, 18, 145.

(46) Wagner, M. H. J . Rheol. 1994, 38, 655.

(47) Vilgis, T. A.; Boue, F. J . Polym. Sci., Part B: Polym. Phys. 1988, 26, 2291.

(48) Edwards, S. F. Polym. J . 1985, 17, 271.

(49) Ngai, K. L.; Roland, C. M. Macromolecules 1994, 27, 2454.

(50) Ngai, K. L.; Roland, C. F.; Yee, A. F. Rubber Chem. Technol. 1993, 66, 817.

(51) Higgins, J . S.; Ma, K.; Hall, R. H. J . Phys. Chem. 1981, 14, 4995.

(52) Oeser, R.; Ewen, B.; Richter, D.; Farago, B. Phys. Rev. Lett. 1988, 60, 1041.

(53) Duering, E. R.; Kremer, K.; Grest, G. S. Phys. Rev. Lett. 1991 67, 3531; Macromolecules 1993, 26, 3241.

(54) Everaers, R.; Kremer, K. Macromol ecules 1995, 28, 7291.

MA960189S 